\title{
Prevention and treatment of venous thromboembolism
}

\author{
Barbara Casadei $^{1 *}$ and Francesco Cosentino ${ }^{2}$ \\ ${ }^{1}$ Division of Cardiovascular Medicine, Level 6, West Wing, John Radcliffe Hospital, Oxford OX39DU, UK; and \\ ${ }^{2}$ Cardiology Unit, Department of Medicine, Solna, Karolinska Institutet, Karolinska University Hospital, \\ 17176 Stockholm, Sweden
}

Medical researchers are typically a fascinating mix of curiosity, creativity, and tenacity (a seemingly limitless supply of tenacity). And the European Society of Cardiology (ESC) has been proud to support many of their efforts through the years.

One of the most significant funding opportunities we have spearheaded is the ESC Grants for Medical Research Innovation, an ambitious programme sponsored exclusively by Boehringer Ingelheim. It was created to foster innovative research in thromboembolic disease, which now kills one in four people. There is an urgent need for new insights into the disease process, and we were astounded by the quantity and quality of proposals we received from grant applicants.

From the 554 applications, the programme's scientific committee eventually selected five researchers in Europe to receive up to $€ 400000$ each. In the following pages, you will be able to appreciate their work.

While we celebrate their success, we are also reminded, yet again, of the mounting challenges facing many scientists today. A recent survey by the Wellcome Trust-the largest of its kind involving $>\mathbf{4 0 0 0}$ researchers the world over-paints a disturbing picture of the culture in which they work (www.wellcome.ac.uk/research-culture).

While most describe themselves as passionate and proud of what they do, only $29 \%$ feel secure in their jobs. No wonder. The survey suggests most (63\%) have witnessed bullying or harassment in the workplace, but only about a third of respondents feel comfortable speaking up. Just over half (53\%) have sought or wanted to seek professional help for depression or anxiety.

This has to change. You are reading this supplement because you value innovative research. The onus is on useach and every one of us-to create a more supportive and inclusive work environment for researchers. We need to find new ways to ensure more men and women, like those represented in these pages, can thrive and apply their talents to the fullest. We will all reap the benefits.

\section{Funding}

This paper was published as part of a supplement supported by an educational grant from Boehringer Ingelheim.

Conflict of interest: B.C.'s research is funded by the British Heart Foundation, the UK National Institute for Health Research and the EU Horizon2020 Programme. She has received in-kind research support (free assays and free ECG monitors) from Roche Diagnostics and iRhythm, respectively. F.C. research is funded by the Swedish Research Council, the Swedish Heart and Lung Foundation, the King Gustav V and Queen Victoria Foundation and has received advisory board/speaker fee from Merck, Novo Nordisk, Pfizer, and Lilly.

${ }^{*}$ Corresponding author. Email: barbara.casadei@cardiov.ox.ac.uk 